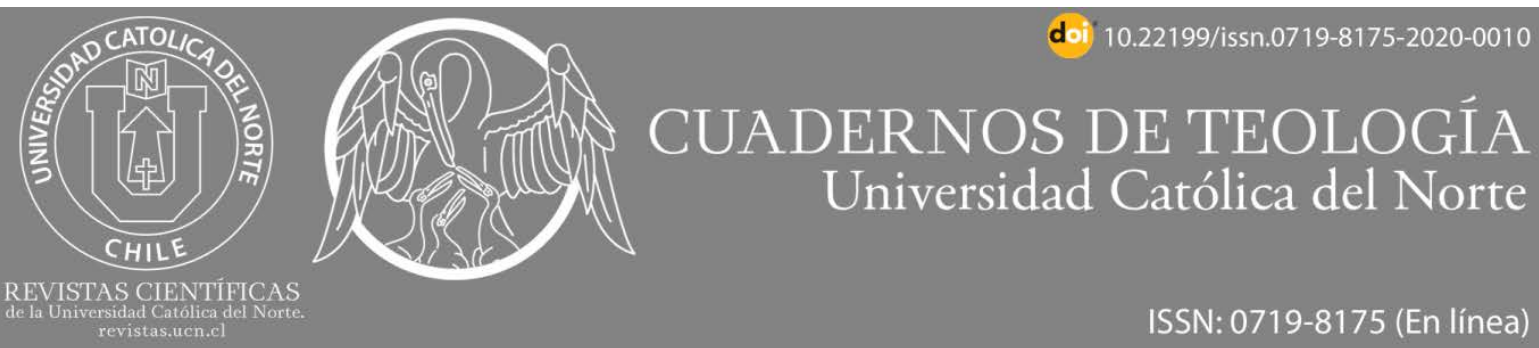

\title{
Finalidad y logro de la educación teológica en un mundo plural: una mirada reflexiva en caso de estudio
}

\section{Purpose and achievement of theological education in a plural world: a thoughtful look at the case study}

\author{
Leonardo Rojas Cadena' (D) orcid.org/0000-0001-6426-031X \\ ${ }^{1}$ Pontificia Universidad Javeriana de Cali, Colombia. Docente. Depto. de Humanidades. Maestría en Desarrollo \\ Humano, U. de San Buenaventura, Cali. \\ - Irojas@javerianacali.edu.co \\ (cc) BY
}

\section{Resumen:}

La formación teológica ha perdido prestigio en el mundo universitario frente a la racionalidad cientificista e individualista que se ha impuesto en la modernidad. Conseguir replantear su finalidad, y su aporte en dicho contexto, permite ubicar los procesos de formación y reconfigurar los medios de aproximación del saber teológico a los contextos educativos. Poder identificar el logro de acuerdo con las finalidades que le impone el contexto, permite juzgar si la mediación realizada de manera didáctica es la adecuada para dichos fines. A partir de la fragmentación de un estudio de caso se busca presentar la reflexión que brota de los datos acerca de las prácticas de la enseñanza de la teología en una universidad de inspiración católica, sus logros y sus desafíos confrontados con las percepciones de los aprendientes del saber. La propuesta de este artículo pretende reflexionar desde los resultados obtenidos acerca de la relación que se establece entre el contenido teológico, el conocer y el saber, pero particularmente entre el conocer y el saber, como proceso de significación y sentido de vida..

Palabras Clave: Formación teológica; Universidad; Mediación pedagógica; Pluralismo.

\begin{abstract}
:
Theological training has lost prestige in the university world in the face of the scientific and individualistic rationality that has prevailed in modernity. Being able to rethink its purpose and its contribution in said context allows to locate the formation processes and reconfigure the means of approaching theological knowledge to educational contexts. Being able to identify the achievement according to the purposes imposed by the context, allows one to whether the mediation carried out in a didactic way is adequate for those purposes. Based on the fragmentation of a case study, the aim is to present the reflection that emerges from the data about the practices of theology teaching in a Catholic-inspired university, its achievements and, its challenges confronted with the perceptions of the students of the to know. The proposal of this article aims to reflect from the results obtained about the relationship that is established between the theological content, knowing and knowing, but particularly between knowing and knowing, as a process of meaning and meaning of life..
\end{abstract}

Keywords: Theological formation; University; Pedagogical mediation; Pluralism. 


\section{Introducción}

La reflexión que se quiere abordar en este texto hace referencia al sentido y significación de la enseñanza teológica en el mundo contemporáneo, en un contexto específico regional en Colombia. Abordar este proceso implica mirar el fenómeno desde su finalidad en contextos de pluralidad realizado por las universidades confesionales, y así poder establecer las prácticas y logros de la misma en sus procesos de enseñanza y aprendizaje. Estas preocupaciones movieron la investigación "Prácticas de enseñanza en la formación teológica en contexto universitario. Estudio de caso múltiple en universidades de fundación e inspiración católica de Bogotá y Cali" la cual permitió indagar, no solo por su realidad en el mundo universitario en contextos específicos, sino a la vez, explorar acerca de las practicas pedagógicas en relación con su finalidad formativa lo que permite a las instituciones en sus procesos de mejora continua, revaluar, mejorar o reafirmar dichas prácticas.

El ejercicio de esta investigación buscó identificar los contenidos, las prácticas y evaluaciones en la formación teológica, lo cual permitió a partir de categorías como concepciones y significados, intencionalidades, actitudes y percepciones, analizar las "prácticas de enseñanza", mirar los modelos pedagógicos que subyacen a las mismas, inferir su coherencia, consistencia y poder generar algunas conclusiones indicativas sobre el tema. Las universidades objeto de estudio fueron: Pontificia Universidad Javeriana (Bogotá), Universidad de La Salle (Bogotá), Pontificia Universidad Javeriana (Cali) y, Fundación Universitaria Católica Lumen Gentium -Unicatólica- (Cali). Este ejercicio nos permite poder responder de alguna manera a la inquietud sobre si la forma en que se enseña la teología (prácticas y enfoque pedagógico) responden a la finalidad de su presencia en el mundo universitario contemporáneo.

En este sentido, utilizaremos los resultados de la investigación de casos, que permite, por los datos recogidos y sistematizados, una aproximación a la problemática propuesta en una de ellas (Pontificia Javeriana-Cali).

Para lograr el objetivo de este texto, se buscará contextualizar la situación desde una serie de presupuestos que ubican la reflexión, luego se hace necesario mirar el significado y finalidad de la formación teológica en el contexto, para luego presentar el impacto de las prácticas de enseñanza según finalidades de la institución confesional. Se hará una reflexión pedagógica desde los resultados del informe de investigación y se confrontará con las percepciones de los aprendientes para generar unas conclusiones finales.

\section{Presupuestos de comprensión}

Aunque no es fácil analizar el lugar de la teología en la universidad, ya que en Colombia no existe una tradición teológica y universitaria muy extendida, se recurrirá a la tradición para su contextualización.

Históricamente la Teología, durante siglos, fue considerada la más importante de las disciplinas universitarias; debido al proceso de secularización vivido en el S.XIX y que se 
heredó en el mundo educativo en América Latina en el Siglo XX, perdió su prestancia social, pero más allá de mostrar cómo se llevó a cabo este proceso, está la pregunta por el puesto que ocupa la teología y si merece ocupar algún puesto en el mundo universitario, lo cual nos pone en un doble plano: epistemológico y político. De alguna manera el tema obliga a plantear el "carácter científico" de la teología y también la "naturaleza" de la universidad en su búsqueda, y comunicación del saber. Ambos temas no están desconectados, sino que se refieren uno al otro.

Los desarrollos ideológicos, desde los cuales se construye la modernidad, en sus diferentes etapas ponen en juego tanto a la tradición individualista liberal, como la tradición antrópica de alteridad que revindica desde el personalismo y otros desarrollos conceptuales, la tesis clásica del carácter social e intersubjetivo del ser humano, no de simple coexistencia (como colonia de champiñones - Hobbes) sino de con-vivencia donde el ser humano se humaniza en sociedad y nunca al margen de ella ya que, desde los complejos de socialización, se logra identidad y a la vez constituye el bien común como espacio de desarrollo de esas individualidades. Se trata de poner presente a la defensa a ultranza de la libertad individual abstracta, la societalidad del ser humano y sus dimensiones que el estado moderno debe tener en cuenta.

No es ajeno al hecho cultural (como hecho) que su existencia es un proceso lento de sedimentación de acontecimientos en la propia historia que permite ser, en gran medida, desde lo que se fue y se vivió; lo cual incluso puede ser trágico para algunas naciones. Los contenidos axiológicos y sus referentes conceptuales ideológicos brotan y se gestan en el pasado, y no pueden ser simplemente ignoradas pues constituyen a los humanos en contexto desde su cultura. (Ningún significado cultural brota de la nada).

En este contexto se infiere que así un estado se declare no confesional, y una sociedad se declare pluralista, no pueden ignorar e invisibilizar los rasgos y aportes de significación religiosa de una tradición que, como el saber Teológico, tiene en el legado cultural e identitario de un pueblo. Donde dicha racionalidad, Teológica, es propia de la racionalidad de occidente. Pero, aun así, políticamente parece no ser correcto apreciar la tradición.

La Teología ha perdido prestigio en las universidades, en oposición a la mentalidad cientificista moderna que se instala en las aulas e incluso en la opinión pública. Ha sido un proceso de entronización de la ciencia empírica como modelo de la experiencia humana, que desvirtúa cualquier otra forma de experiencia y significación que no brote de la razón Esta verdad, la verdad de la ciencia, es la única que nos hará libres. En este contexto la teología ha perdido prestigio en su afán de competir en el mismo plano de la cuantificación de la racionalidad empírica.

Aunque en este contexto existen avances desde la epistemología y propuestas ${ }^{1}$ desde las cuales se revalúa el cientificismo absolutista, dándole un lugar entre los saberes y

\footnotetext{
1 Kuhn, por ejemplo, ha mostrado que los cambios de paradigma científico obedecen a factores que nada tienen que ver con la lógica de la investigación científica; Feyerabend afirma que el método científico es incapaz de explicar su propia racionalidad («la ciencia es un cuento de hadas, y encima falso»); Popper sostiene
} 
compresiones humanas de sí mismo y su contexto y que, por lo tanto, la ciencia no puede emitir juicios sobre otros saberes sin estar en su plano experiencial. Es preciso reivindicar y hacer presente la legítima diversidad de las formas de la experiencia humana.

Desde estos contextos descritos se plantea la pregunta por la presencia de la Teología en las Universidades, o es que en ellas ¿solo hay cabida para las disciplinas estrictamente técnicas y científicas? En medio de este debate se encuentran también las humanidades y sus saberes que propenden por la humanización de las sociedades en contra del profesionismo técnico propio del cientificismo.

Así como se decía que estudiamos ética, no para saber más, sino para ser mejores, esto se le puede aplicar a todos los saberes en la medida en que toda vocación intelectual nace del sentimiento de responsabilidad por nuestro propio ser y obrar en el mundo. Siendo la responsabilidad una de las características de humanidad, al hombre responsable no le es indiferente nada, ningún saber, ya que lo que está en juego es el sentido de su existencia individual e incluso de la existencia de la humanidad en su conjunto.

La era de la tecnociencia que obnubila a todo militante en ella, impide ver sus orígenes, raíces y finalidad, poniendo en muchas ocasiones en juego la dignidad de las personas, y no en vano este concepto de persona tiene su origen en los debates Teológicos que orientaron gran parte del desarrollo del cristianismo y que hoy, desde una mirada amplia e incluyente, busca la rehumanización del mundo como sistema (Francisco, 2015).

Estos, y seguramente otros muchos, son los presupuestos que ayudan a ubicar el debate de la educación confesional en contextos latinoamericanos, como el colombiano, donde existen voces que sostienen que, las Universidades confesionales, deben ser excluidas de los sistemas de educación, y que tanto la formación en humanidades, como la teológica, son una pérdida de tiempo en la educación universitaria que requiere de seres libre con habilidades técnicas que les permitan desarrollar el mundo fruto del magnetismo que produce el poder, siempre creciente, de los saberes científico-técnicos y embebidos en la ideología del progreso ilimitado.

En este sentido, en una atrevida primera conclusión, se podría afirmar que, así como la universidad nace en el seno de la Iglesia y logra su desarrollo, igual se podría argumentar, que sin la teología no hay universidad, y, por otra parte, que sin universidad no hay teología. Y que, la Universidad Católica, puede y debe custodiar la "esperanza de la razón" (Cf. Ladriere, 1996) y que allí esta su vocación más profunda para el presente y el futuro.

que la ciencia no puede ir más allá de la formulación de conjeturas, las cuales serán rechazadas y sustituidas por nuevas hipótesis, que sufrirán el mismo destino — dicho de otro modo: la verdad de que se jacta el cientificismo es siempre una verdad interina, nunca definitiva. 


\section{Teleología de las Universidades confesionales y formación teológica.}

La reflexión que se planteará hará referencia a las universidades de fundación e inspiración católica, objeto de la investigación ${ }^{2}$ que da origen a este texto, (Pontificia Universidad Javeriana - Cali, Bogotá; Universidad Lumen Gentium Cali, Universidad de la Salle Bogotá), en el contexto del debate por la educación y la formación en contextos de pluralidad. Uno de los argumentos más repetidos es que, aunque logran calidad académica, no están al servicio del interés público y representarían una forma de proselitismo en favor de las ideas católicas.

La libertad de cultos, expresada en la constitución, como la pluralidad generan son sectores de exclusión que impiden apreciar, en su globalidad, el aporte de las universidades confesionales al concierto universal y educativo de un país, de una región. Es claro que ninguna institución educativa es ajena a una serie de principios que fundamenta su accionar y genera identidad; en términos generales todas ellas buscan que sus estudiantes desarrollen actitud reflexiva, dialogante y crítica, que luchen por la justicia y la equidad; personas con sentido ético, cívico, de respeto a la diferencia y de solidaridad social; donde las instituciones se comprometen con el desarrollo de la región y el país, todo esto a partir de sus funciones sustantivas.

De aquí brota naturalmente la pregunta sobre los caminos formativos que las instituciones toman, para lograr generar identidad y diferencia en su aporte social. El profesor Zuleta hace evidente que la contribución de la Universidad católica es fundamental en momentos de crisis social de todo tipo y en la formación del sentido humano integral (Zuleta 2013. 284). Esta formación de sentido logra confrontar los sistemas de pensamiento y las teorías evitando la homogenización de la Universitas como un simple taller, hacedora de profesionales con perfiles productivos laborales. El criterio más alto del espíritu de la universidad estaría en propiciar "un lugar de sentido donde el encuentro y la apertura relacional de respeto e integridad, fortalezcan el nivel de conciencia, la integridad y el respeto por la vida propia y la de los otros. Donde los aprendizajes, competencias y técnicas estén al servicio de la vida" (Zuleta 2013, 283). La misión de la Universidad católica es, entonces, poner en diálogo el mundo de la ciencia con la experiencia cristiana, en todas los aspectos y conversaciones intelectuales que le atañen desde el objeto propio de su desarrollo teológico.

Juan Pablo II, en la Constitución apostólica Sapientia Christiana (1979), aborda la tradición y modernidad de la pedagogía teológica, instando a trascender el marco académico para inspirar de sentido cristiano la realidad vital del hombre y la cultura. Parafraseando al Pontífice, no se debe privar, de los avances y desarrollos teológicos, a una sociedad que requiere generar sentidos más amplios e íntegros de humanidad, es necesario, por tanto, que los cultivadores de las ciencias sagradas, mientras llevan a cabo su "...deber fundamental de conseguir, mediante la investigación teológica, un conocimiento más profundo de la verdad revelada..."(Juan Pablo II, 1979, 3)., se mantengan en relación con los

\footnotetext{
2 "Prácticas de enseñanza en la formación teológica en contexto universitario. Estudio de caso múltiple en universidades de fundación e inspiración católica de Bogotá y Cali" Investigación realizada en los años 2019-2020.
} 
estudiosos de las otras disciplinas, sean creyentes o no, e intenten entender bien y valorar sus afirmaciones, "...y juzgarlas a la luz de la verdad revelada". (Juan Pablo II, 1979, 3).

Ya la tradición universitaria confesional desde el siglo XIX, y aún desde antes, ha puesto de presente en sus aulas la importancia de defender la dignidad humana y derechos del hombre (posteriormente derechos humanos), desde una visión antrópica cristiana; esa búsqueda es por la libertad ilustrada de la conciencia y la libertad responsable que le es propia, al igual que el diálogo respetuoso y una promoción de la justicia. Hoy, más que nunca, es claro que, aunque el objeto de la Teología como saber trasciende el plano cultural, acontece en la realidad humana e histórica inspirando el sentido trascendente de la realidad humana, siendo la tradición magisterial de la Iglesia y la sagrada escritura sus fuentes que entrelazan la diversidad de su desarrollo. Las universidades y facultades eclesiásticas no están para servir exclusivamente al Magisterio de la Iglesia (S.C. 1979), su esfuerzo y desarrollo debe trascender el ámbito de la formación teológico-académica para incardinarse con fuerza en la realidad existencial.

Se quiere dejar constancia que, el contexto plural y dinámico de la sociedad contemporánea, le implica a la universidad católica una exigencia de diálogo entre la praxis científica con los saberes humanos y teológicos, es decir, hacerse cargo de la creación de espacios de encuentro y articulación de lenguajes, que permitan ampliar las miradas de sentido en forma holística e integral; un diálogo interdisciplinar y abierto a los avances y posibilidades de la ciencia a la humanización del mundo en relación con la verdad de la fe, en un compromiso moral que brote de la Caridad.

En este orden de ideas, se puede afirmar que la formación teológica, en las Universidades con inspiración cristiana católica, está alrededor de construir significados de sentido humanos, holísticos abiertos a la trascendencia como reto pedagógico en contextos plurales. La educación en sus diversas propuestas formativas confluye en algunos puntos: el cultivo de una sensibilidad humana profunda que dé, a la vez, empatía y capacidad de discernimiento, que ayude a romper con la 'mismidad egocéntrica' que nubla la conciencia colectiva y sentido de sistema en un sistema biótico-ecológico y social, en la búsqueda de la calidad humana y su sentido de trascendencia, empoderando desde la libertad inspirada en el amor con profunda responsabilidad histórica.

En consecuencia, la tarea de la teología es poder enriquecer y fortalecer la vida social, propiciar diálogo, convivencia y reconocimiento más que enseñar la religión (es) como un producto en oferta, no se enseñan formulaciones, se enseña a pensar teológicamente, además se colabora con la Iglesia a conocer mejor la fe que ella misma confiesa (Treek Nilsson 2015). Esto significa, pedagógicamente, ayudar a un vasto público a desarrollar un pensamiento crítico sobre el objeto que se estudia y sobre la forma en la que cada cual se sitúa frente a él.

La Teología en las universidades se juega su valor en el espacio público, ya que el creyente (de cualquier religión) es un ciudadano con derecho a ser respetado y oído en su intento de traductibilidad. Las universidades no pueden renunciar a formar personas que 
examinan su pensamiento y el de los demás, considerando justo que otros también les hagan ver sus propios límites:

A quienes sueñan con una doctrina monolítica defendida por todos sin matices, esto puede parecerles una imperfecta dispersión. Pero la realidad es que esa variedad ayuda a que se manifiesten y desarrollen mejor los diversos aspectos de la inagotable riqueza del Evangelio. (Francisco, 2013, 40)

Por ello que, el ciudadano no creyente, también realiza un esfuerzo en orden a no descartar, por principio, los aportes que provengan de lo religioso, siempre que hayan sido traducidos de manera tal que resulte posible entablar una conversación razonable (Habermas, Taylor, Butler, y West, 2011).

La enseñanza de la teología se convierte entonces, en la mejor forma de mostrar como el cristianismo cree en un Dios, que se ha hecho legible en la historia y audible en el mundo que exige estar en contacto con los movimientos sociales para discernir qué, de esos movimientos, es compatible con el Evangelio y qué no lo es, en un profundo compromiso con la vida de su audiencia y el objeto de su reflexión. Pero la pregunta que se sigue es ¿cómo se está generando este diálogo teología, ciencias, realidad? Según lo mostrado hasta ahora, en el contexto Universitario, en las instituciones de inspiración católica ¿Logran su objetivo?

Es aquí, y sin pretensión de universalizar la respuesta, donde la investigación nos genera pistas sobre las prácticas y logros de la enseñanza teológica en las universidades, en el caso que nos convoca en este escrito: la Pontificia Universidad Javeriana Cali.

\section{La formación teológica como una práctica de enseñanza desde la perspectiva de la mediación pedagógica}

Después de haber presentado la ubicación y sentido de la teología en la universidad, se hace necesario presentar, en el quehacer pedagógico, las prácticas de enseñanza con la pretensión de explorar cómo entender las prácticas de enseñanza en la teología, y así poder analizar los elementos que están en juego en esta investigación.

Desde lo presentado en los apartes anteriores, surge como hipótesis que la Teología en la Universidad es un saber situado que requiere de una pedagogía que responda a esta característica. Cuando se indaga por las teorías pedagógicas que tratan de orientar el vínculo entre la institución educativa y la vida, se hace necesario realizar una aproximación a los elementos que del proceso de formación: Donde la Actividad educativa por parte del docente (manifiesta o encubierta) se refiere a la enseñanza; las actividades de aprendizaje por parte del Aprendiente (manifiestas o encubiertas) hacen referencia al Aprendizaje, y el Contenido de los saberes el cual está en medio de las actividades docentes y aprendientes. (Díaz Barriga, 2006). Se entenderá «sujeto aprendiente» como la articulación que van armando el sujeto cognoscente y el sujeto deseante, sobre el organismo heredado, construyendo un cuerpo, siempre en interacción con otro (Conocimiento-Cultura...) y con otros (padres, maestros, medios de comunicación). 
El aprendiente se sitúa en la articulación de la información, el conocer y el saber, pero particularmente entre el conocer y el saber. Proceso en el cual intervienen inteligencia y deseo. (Fernández 2007, p. 73).

Autores como Francisco Gutiérrez y Cruz Prado (2015) ubican el quehacer pedagógico en: enseñanza-aprendizaje, contenido desde la mediación y facilitación del contenido (contexto, didáctica, metodologías, actividades). Es por ello que, el enfoque en la recolección de información se ubica en estos puntos: el enseñante, el aprendiente y el contenido mediado.

En favor de lograr indagar por las practicas pedagógicas, se inició un trabajo que permitiera inferir en dónde se encontraba el acento y la fuerza del modelo pedagógico utilizado, en palabras de Díaz Barriga (2006) "metáforas educativas" como expresión de las racionalidades que la sostiene, el tipo de interlocución se establece entre el enseñante y el aprendiente y la fuerza del contenido en esa interlocución desde los intervinientes en el proceso.

Por ello, para lograr el objetivo de la investigación, se presenta en primer lugar el análisis de las muestras en torno a las concepciones, intencionalidades, aptitudes y percepciones de los entrevistados en torno a la categoría Formación teológica. Dicha categoría se entiende, en este estudio, como el universo de intencionalidades y currículo formativo teológico ofrecido por la Pontificia Universidad Javeriana Cali y concretado en planes, áreas y asignaturas. (La sistematización de la información se realizó con la herramienta Atlas Ti, grupos focales, entrevistas y test) ${ }^{3}$.

En el ámbito del enseñante (docente) se manifiesta claridad en cuanto: finalidad de la formación en cuanto apuesta institucional, donde su sentido es amplio en cuanto la apuesta teológica está en función de la vida, y de aportarle a encuentro de sentido de la misma en sus relaciones trascendentes desde lo cotidiano, es una apuesta "humana por la humanidad" (Grupo Didaskalia, 2019, pp. 5-7). Esta primera aproximación nos conecta con uno de los sentidos de la teología en el mundo universitario y es crear espacios de diálogo.

Aunque no está en juicio la apuesta institucional pedagógica, si es importante hacer notar que, el modelo pedagógico ignaciano, genera una impronta tanto en los docentes como en los estudiantes, confirmando en los enseñantes que existe una dimensión humanista, que se propende por una formación y acompañamiento integral, que es abierta al pluralismo y no es una teología dogmática en el sentido tradicional, lo que la hace dialogante.

Ahora bien, si la mirada se hace desde el aprendiente el resultado que arroja la sistematización de la Investigación, resalta la experiencia que pide ser significada desde la vida de los aprendientes, el significado no está dado, se logra descifrar en el tiempo y en el transcurso tanto de la asignatura como de la vida universitaria. Las significaciones se abren a búsqueda de calidad humana, "abrir nuestra mente y nuestra disposición a otro tipo de conocimientos y a entender como el relacionamiento con los demás" (Grupo Didaskalia, 2019,

\footnotetext{
${ }^{3}$ Informe realizado a partir del procesamiento de la herramienta Atlas Ti.
} 
p. 9) en diálogo a la pluralidad; se ve como una oportunidad de potenciar sus profesiones a partir del desarrollo humano. Aparecen por primera vez las prácticas de teología, las cuales son parte de una mediación de contenidos que les "permiten el encuentro personal con realidades de sufrimiento y exclusión" (Grupo Didaskalia, 2019, pp. 10-11) recalcando cómo la experiencia que los cursos brindan los forma en valores (les ayuda a significar la vida para mirar el mundo y tomar decisiones) y les invita a implicarse en la realidad como profesionales y futuros agentes sociales.

Es una apuesta en libertad, "es muy importante ese espacio de reflexión personal y yo creo que la función como agente de socialización que tiene la reflexión personal frente a la espiritualidad, a la trascendencia, al proyecto de vida personal" (Grupo Didaskalia, 2019, pp. 13). Claro es que hay una apuesta institucional desde la identidad de la institución, pero es también interesante su apertura y diálogo desde esa identidad. Hay claridad que se "generan espacios significativos, donde ellos puedan tener dos cosas: primero: la capacidad igual de entender las religiones y el rol de las religiones, las prácticas de espiritualidad, pero también un espacio donde ellos mismos puedan hacer su reflexión y generar una postura crítica personal" (Grupo Didaskalia, 2019, p. 13).

Estos primeros resultados llevan a inferir que existe la intención que el aprendizaje que brinda la teología es significativo, es decir que busca que el estudiante encuentre sentido a lo que hace. El énfasis está en potenciar las capacidades del aprendiente para que logre su desarrollo; es aquí importante mostrar cómo se logra cambiar los procesos de interlocución en la educación, ubicando las claridades institucionales de su identidad en el proceso.

Como se puede ver, existe una interesante diferencia entre enseñanza y aprendizaje, pero el resultado del informe permite inferir cómo el aprendizaje está volcado en la experiencia del ser, que está inmerso en la tarea de construirse y recrearse abriéndose y apropiándose tanto de la ciencia como del mundo. Aunque es claro que, tanto enseñanza como aprendizaje, están en función de promover el proceso educativo, es importante señalar que más que solo en la enseñanza, sino en el proceso educativo permite mediar los conocimientos de tal manera que logren ser significativos para el aprendiente.

O se propone una enseñanza con sentido o se impone el sin sentido de la educación. Este dilema que enfrenta la pedagogía latinoamericana, también se hace presente en el mundo de la formación teológica, es donde contenidos y significaciones se hacen visibles, es cuando la mediación de los mismos se hace importante para que conecten sentidos: el que ofrece el contenido con la vida contextuada del aprendiente. Dar sentido a lo que se hace: ofrecer oportunidades para que el estudiante de sentido a lo que se hace, para que pueda compartir sus sentidos con otros estudiantes, para que logre impregnar del propio sentido las prácticas cotidianas tanto en la escuela y la familia como fuera de ella. El sentido puede llevar a encontrar el sin sentido de las acciones pedagógicas de la escuela.

En un estudio precedente, sobre "Teología: su epistemología y los nuevos paradigmas" (Agudelo, Carrasquilla y Rojas, 2004) muestra cómo, desde la especificidad del contenido de la Teología y su abordaje, puede lograr los virajes que desde la práctica de enseñanza se 
propondrían para realizar un énfasis en el aprendizaje contextuado y significativo. Los autores proponen analizar la estructura propia de la teología, para posibilitar la eficacia de su enseñabilidad en la formación universitaria, pues la enseñabilidad está en relación con la posibilidad de entender las racionalidades subyacentes y las formas a partir de las cuales se puede ir generando acercamiento, tanto con la vida como con otros saberes. La exigencia siempre estará alrededor de lograr hacer de los cursos espacios vivenciales y prácticos (Grupo Didaskalia, 2019, p. 26) en los cuales los saberes también estén atravesados por las cuestiones y aportes teológicos.

Ya que la teología debe estar en diálogo con los problemas del hombre y su sociedad, que se fundamente en el Evangelio interpretado con método riguroso y que se correlacione con la vida y sus exigencias éticas; es interesante notar cómo los grandes temas son recordados en relación con las aproximaciones humanas y ecológicas que requieren ser atendidas en nuestra cotidianidad. Pareciera existir una dicotomía entre propuestas desde la teología y la vida académica profesional, lo cual hace que se falsee uno de los fines de la formación teológica y es el diálogo con los demás saberes. Profesores y estudiantes destacan que la mediación de los contenidos es creativa, buscando distintos recursos que faciliten el aprendizaje; la teología debe estar "abierta" y en permanente crisis, lo que supone una constante búsqueda: "una teología siempre en proyecto y en estado de reforma" (Agudelo et al. 2004, p. 459).

En este sentido, la pedagogía promociona el aprendizaje a través de los recursos puestos en juego en el acto de la enseñanza, que lo convierten en un acto educativo. La tendencia que se presentan busca que se tengan en cuenta la realidad que se quiere transformar y el futuro que se desea construir. Se propone desde un horizonte de participación, creatividad, expresividad y relacionalidad. Definitivamente el elemento que conecta a los estudiantes con un aprendizaje significativo son las prácticas de teología, las cuales son recordadas significativamente por los estudiantes. (Grupo Didaskalia, 2019, p. 32).

Siendo el contenido trabajado en la teología de tipo simbólico, ya que expresa con mayor profundidad la experiencia de Dios desde la condición humana (Heb 4, 12) y que experiencia no es unívoca, sino polisémica; se podría afirmar, expresado en forma sencilla, no simplista, que se trata de una experiencia, donde la dificultad pedagógica es que las experiencias no se pueden transmitir, o enseñar, pues requieren de una significación construida desde el sentido del sujeto, la posibilidad de mediación es más compleja para no terminar en una estructura de contenido vacía de sentido en el aprendiente.

Los estudiantes reconocen que en los cursos de teología puede han podido tener un aprendizaje u significativo mediante la reflexión y la experiencia dado que los docentes implementan estrategias variadas para validar el alcance de los objetivos. Es valorado por los estudiantes el hecho que la evaluación no se centre en exámenes parciales y que las lecturas, que en muchos casos son densas para estudiantes no teólogos, sean abordadas con distintas estrategias como por ejemplo los mapas conceptuales. (Grupo Didaskalia, 2019, p. 35) 
De lo anterior también se puede evidenciar el reto que supone significativamente el lenguaje que se utilice, "lo que no se hace sentir no se entiende" decía Simón Rodríguez ${ }^{4}$, y lo que no se entiende no interesa. Si queremos dar sentido a lo que hacemos (sentido, intuición, emoción) se ha de tener encinta la dimensión subjetiva.

El sentido no se traspasa, el sentido se construye, se hace y se rehace en un proceso de descubrimiento y enriquecimiento permanentes. No son los conocimientos que se transmiten los que dan sentido a la vida. El sentido se entreteje de otras maneras, desde las relaciones inmediatas, desde cada ser, desde los contextos en los cuales se vive desde procesos y relaciones significativas.

Sin embargo, esta experiencia de fe en cuanto enseñable a los otros siempre será parcial y provisional. porque no podrá agotar completamente el misterio de Dios comunicado en la persona de Jesús de Nazaret, como nos lo recuerdan los autores (Agudelo et al., 2004). Aquí es necesario recordar una constatación psicológica aplicada a la pedagogía realizada por King, citado por Santrock $(2002,349)$ y es que el conocimiento es situado "Se genera y se recrea en contextos, está anclado y conectado con el lugar donde se construyó", luego se podría deducir que el estudiante desee saber, o sepa, y valore a partir de sus conocimientos aptitudes y disposiciones en una confrontación con sus contexto familiares y sociales. Esto es lo que permite una comprensión de los contenidos curriculares vinculado a los saberes personales, es decir que generen una relación con un uso funcional del mismo. Que articule de manera sensible con los problemas de su mismo contexto. (Díaz Barriga, 2006, p. 12).

Lo que se está planteando es que, para hacer teología hoy y buscando sea significada en el ámbito del aprendizaje, es necesario considerar que ella es fundamentalmente una hermenéutica crítica de la acción de Dios en la propia existencia y exige como respuesta una fe contextuada. (Agudelo et al., 2004, p. 459). Que en la búsqueda de sentido en el aprendiente no puede quedarse en una "la voz oficial de una iglesia" sino que su búsqueda está en articular, razonablemente, la voz profética de Dios en medio de una comunidad concreta para jalonar la historia hacia su propia superación en Dios. (Agudelo et al., 2004, p. 459). Se hace imperativo el diálogo con el mundo plural (Cf. p. 464) teológico "el inclusivismo y el pluralismo" como elementos fundamentales y escenario de debate.

Siguiendo el texto de Frida Díaz, (enseñanza situada) se hace necesario recuperara el contexto y la vida en la enseñanza, no importa el nivel que él suponga, pues el aprendizaje está en función de la vida y es aquí:

...donde cobran sentido las propuestas educativas referentes a aprendizaje significativo, autoaprendizaje compartido, donde la meta es la construcción del conocimiento y habilidades adaptativas y cooperativas en la solución de problemas tanto académicos como cotidianos. Constructivismo, herencia que lleva a pensar el aprendizaje como un proceso de construcción de significados cuyo atributo definitorio es su carácter dialógico y socia". (Díaz Barriga, 2006, p. 13)

\footnotetext{
${ }^{4}$ Educador y político venezolano.
} 
Pero, no se puede caer en un ensueño ingenuo, ya que a pesar de este desarrollo pedagógico que data en sus primeras propuestas a comienzos del siglo XX (John Dewey. 1859-1952. Aprender haciendo) y sus desarrollos posteriores ${ }^{5}$ como lo dice el informe de la Dirección General de Educación y Cultura, de la Comisión Europea (2000), las practicas docentes aún se mantienen buscando solo explorar y evaluar conocimientos declarativos. Se busca entonces centrarse en el aprendizaje del alumno y en la formación docente que responda a las concepciones de los actores educativos.

El conocimiento es situado, es parte y producto de la actividad, del contexto y de la cultura en que se desarrolla y utiliza. Es claro que es mediado por agentes educativos a través de los cuales se integran los estudiantes a comunidades de aprendizaje o en prácticas sociales, donde el conocimiento no es una cosa sino un fenómeno social. (Díaz Barriga, 2006, p. 21)

Para el fin que nos ocupa, no se puede olvidar que son los contextos de aprendizaje y enseñanza los que otorgan facilidades o imponen restricciones al desarrollo del proceso. $\mathrm{Y}$, por lo tanto, lo que se buscaría cuando se habla de "prácticas de la enseñanza en la teología" es resolver la pregunta alrededor de las prácticas, modelos y estrategias que permitan transformar e innovar las concepciones y prácticas cotidianas del aula. Donde aprender, hacer y reflexionar en este sentido son indisociables.

Lo que está en juego es una forma de concebir el aprendizaje, el fin educativo de la teología como un deber ser de su enseñanza su papel social, sus funciones. (Díaz Barriga, 2006, p.15).

Es claro, en este momento del texto, que las "prácticas de enseñanza de la teología" están en el mundo del aprender, no como una transmisión-recepción, sino una construcción mediada de significados, que como lo expresa Díaz Barriga (2006) "implica actividades intención, acción y reflexión; donde mente, conducta, percepción, los afectos y la acción integran un todo". (p. 17). También es necesario recordar que el sujeto está siempre abierto al aprendizaje, está receptivo a la información, pero está mediado por el interés y el bienestar, el aprender está en función de la vida, de lograr desarrollo y acoplamiento social vital adecuado (Gutiérrez y Prado, 2015).

\section{Datos expresados desde el aprendiente}

De manera simultánea, como herramienta de recolección de información, se realizó una encuesta de percepción a los grupos de participantes de la asignatura de teología, la cual fue contestada por 387 estudiantes con resultados que, confrontados con los argumentos precedentes, permite formular algunas conclusiones frente al tema que nos ocupa. La intensión fue poner el discurso perceptivo en el aprendiente.

\footnotetext{
${ }^{5}$ Enfoques nacidos del constructivismo y de las perspectivas conocidas como cognición y enseñanza situada, aprendizaje experiencial y enseñanza reflexiva.
} 
Como ya se presentó anteriormente, existe la percepción tanto de la pertinencia como de su sentido formativo:

\section{¿El objetivo de la asignatura es? \\ 387 respuestor}
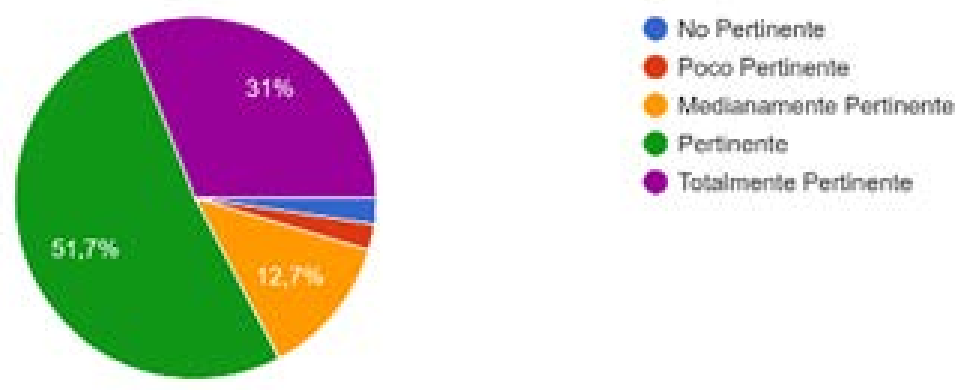

De igual manera lo referente a los temas que permiten el desarrollo de los objetivos de formación son considerados pertinentes en un alto porcentaje.

¿Las temáticas que se abordan en la asignatura son?

387 respuestas

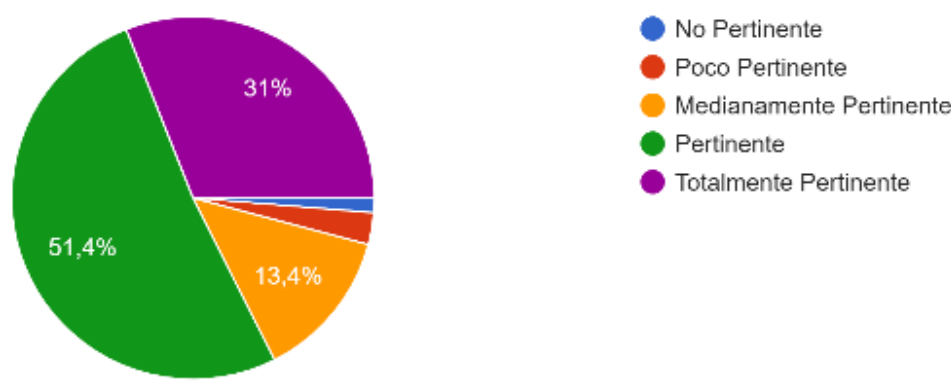

La percepción del tratamiento metodológico de la asignatura, como la conexión con la vida, lograron un alto grado de pertinencia, pero no por ello se descuida la desviación que se presenta la poca y mediana pertinencia, lo cual obliga a buscar y reforzar caminos que las logren.

¿La metodología implementada para el desarrollo de la asignatura es? 387 respuestas

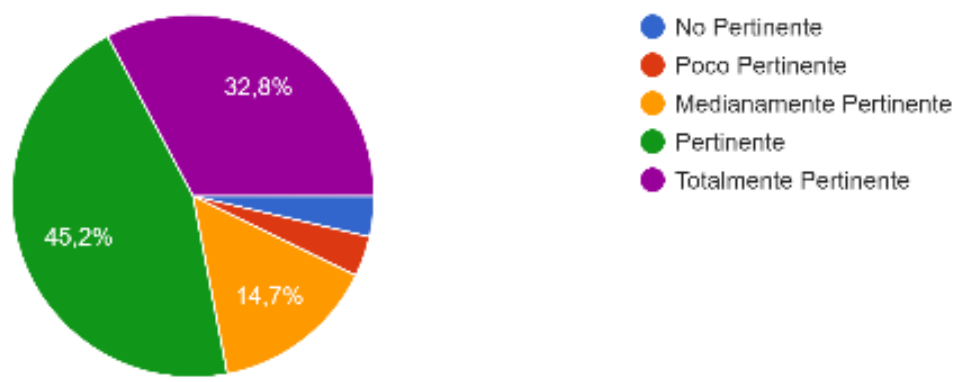


¿La conexión entre las temáticas que se desarrollan en la asignatura y su vida es?

387 respuestas

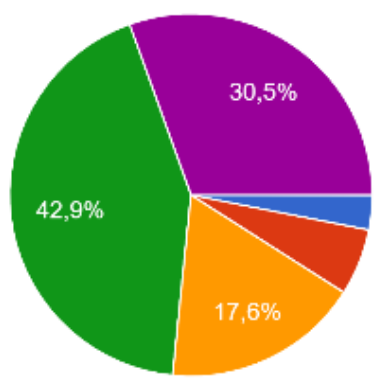

No Pertinente

Poco Pertinente

Medianamente Pertinente

Pertinente

Totalmente Pertinente

¿La conexión que genera la estructura temática unida a la experiencia práctica con las necesidades humanas y sociales del estudiante es?

387 respuestas

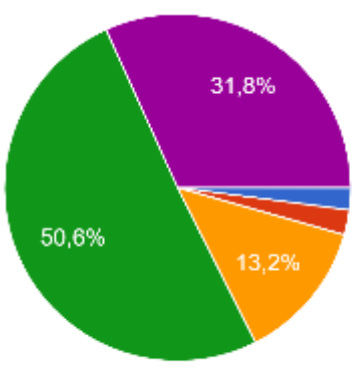

No Pertinente

Poco Pertinente

Medianamente Pertinente

Pertinente

Totalmente Pertinente

Es importante señalar que la contextualización es la que permite la significación de sentido como se explicó anteriormente. Además, en relación con la identidad misma de la institución, se alcanza a percibir fuertemente por los aprendientes el aporte que la teología realiza a la identidad.

¿La formación que se brinda en la asignatura desde la excelencia humana y académica, en la perspectiva del MAGIS ignaciano es?

387 respuestas

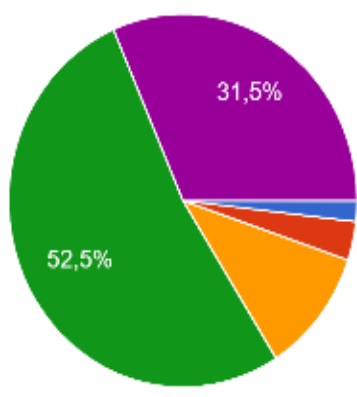

No Pertinente

Poco Pertinente

Medianamente Pertinente

Pertinente

Totalmente Pertinente 
El elemento que se busca identificar es la metodología y tratamiento de los procesos didácticos que sostienen los logros presentados en forma precedente. Se destacan:

¿La metodología, herramientas didácticas y los recursos pedagógicos que implementa el profesor para desarrollar los temas de la asignatura es?

387 respuestas

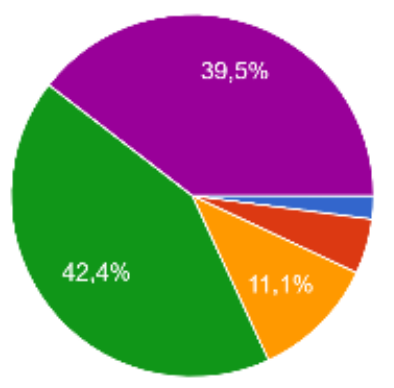

No Adecuada

Poco Adecuada

Medianamente Adecuada

Adecuada

Totalmente Adecuada

La actitud del docente como mediador de los contenidos:

¿La correspondencia entre teoria y práctica, valorada desde las actitudes del profesor es? 387 respuestas

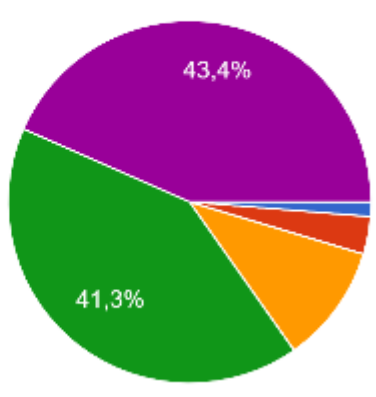

No Adecuada

Poco Adecuada

Medianamente Adecuada

Adecuada

Totalmente Adecuada

El acompañamiento del docente en los procesos de formación del aprendiente:

¿La acogida y acompañamiento que brinda el profesor de la asignatura es?

387 respuestas
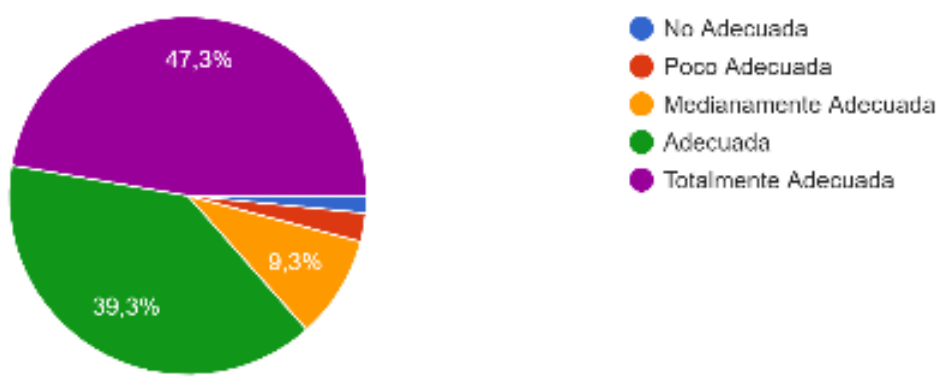

Cuad. teol. - Univ. Catól. Norte (En línea) 2020, 12: e4636 
¿El acompañamiento integral y asesorias académicas que brinda el profesor a sus estudiantes son?

387 respuestas

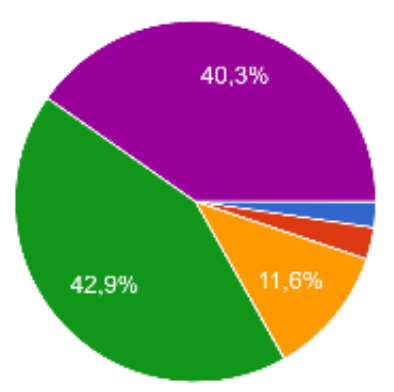

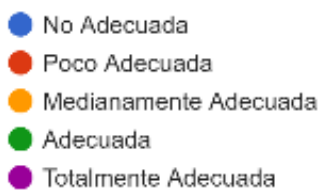

Totalmente Adecuada

Las experiencias "prácticas" (salidas, acompañamiento y conocimiento de comunidades y situaciones que evidencien los contenidos temáticos de las asignaturas) son el punto más importante en la valoración de los estudiantes:

¿Las guias y textos de lectura que implementa el profesor para abordar, problematizar y contextualizar los temas de la asignatura son?

387 respuestas

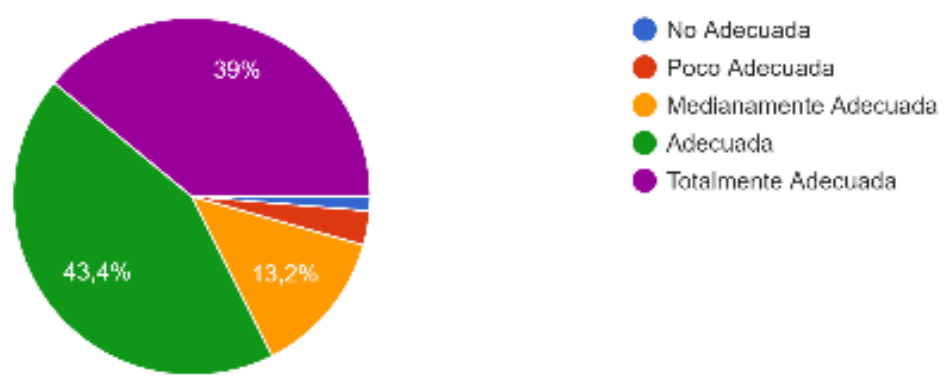

¿La conexión entre el tema desarrollado en la experiencia práctica y las temáticas desarrolladas en la asignatura de Teología l es?

38 sespuestas

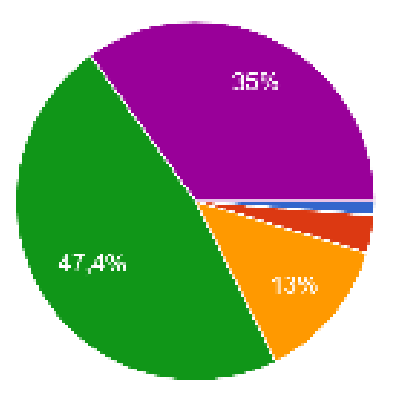

To:almenter Perlimente 
¿La conexión entre la experiencia práctica y el objetivo de la asignatura de Teologia l es?

386 respuestas

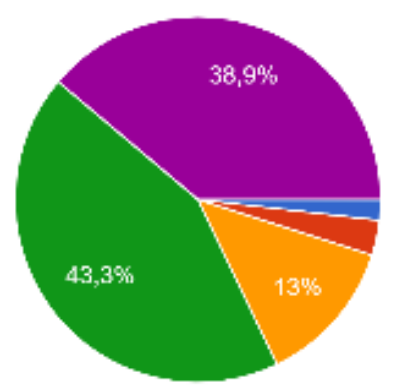

No Pertinente

Poco Pertinente

Medianamente Pertinente

- Pertinente

- Tolalmente Pertinente

Todo este proceso lleva a poder evaluar la actitud desde la percepción del aprendiente en su compromiso con la asignatura, lo cual muestra altos grados de pertinencia en cuanto disposición y trabajo en las actividades propuestas en la asignatura que permiten darle sentido a los contenidos presentados.

¿Mi disposición para el trabajo y la investigación en la asignatura es?

386 respuestas

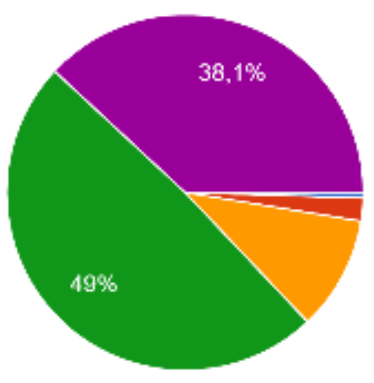

No Adecuada

Poco Adecuada

Medlanamente Adecuada

Adecuada

Totalmente Adecuada

¿Mi responsabilidad para participar activamente en la preparacion y desarrollo de las actividades académicas es?

386 respuestas

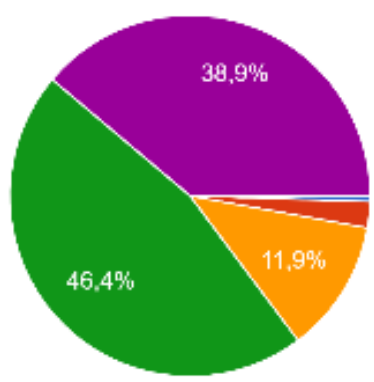

No Adecuada

Poco Adecuada

- Medianamente Adecuada

Adecuada

Totalmente Adecuada 

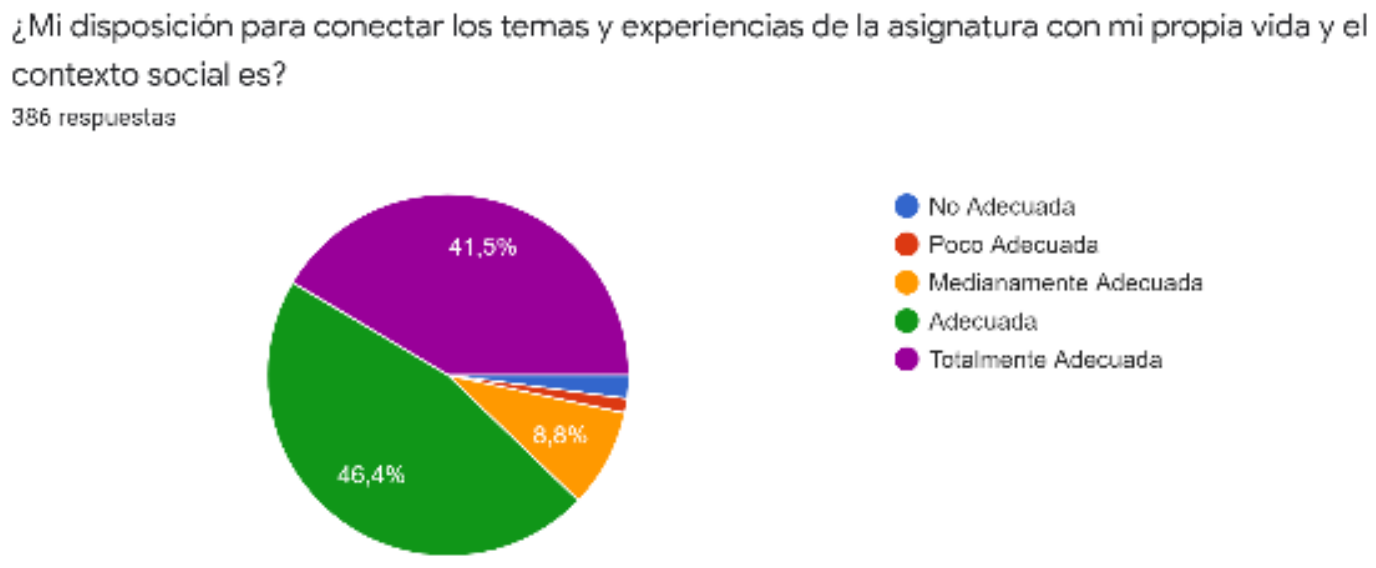

No Adecuada

Poco Adecuada

Medianamente Adecuada

Adecuada

Totalmente Adecuada

No se puede pasar por alto que este proceso no es solo individual, sino que supone una relación con el contexto del curso, en el cual se encuentra el estudiante, cómo el grupo se hace corresponsables de las acciones pedagógicas de aprendizaje.

¿La disposición del grupo para generar conexión entre los temas y experiencias de la asignatura con la formación humana y el contexto social es?

386 respuestas

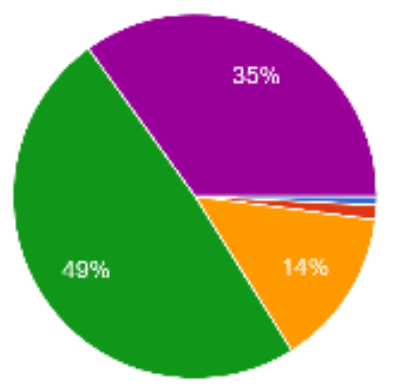

No Adecuada

Poco Adecuada

Medlanamente Adecuada

- Adecuada

Totalmente Adecuada

\section{Conclusiones}

Si se analizan las finalidades en contraste con los logros y hallazgos que arroja la investigación en el caso específico tratado se puede concluir que:

La enseñanza de la teología, en un contexto dinámico de la sociedad contemporánea, permite construir significados de sentido humanos, holísticos abiertos a la trascendencia como reto pedagógico en contextos plurales.

El trabajo realizado fundamentado en la construcción de sentido desde el aprendiente permite el cultivo de una sensibilidad humana profunda, que dé a la vez empatía y capacidad de discernimiento, empoderando desde la libertad, inspirada en el amor, una responsabilidad histórica.

Además, si la tarea de la teología es poder enriquecer y fortalecer la vida social, propiciar diálogo, convivencia y reconocimiento, significa que pedagógicamente se ayuda a 
desarrollar un pensamiento crítico sobre el objeto de estudio de la teología y sobre la forma en la que cada cual se sitúa frente a él.

La universidad no renuncia a formar personas que examinan su pensamiento y el de los demás, considerando justo que otros también les hagan ver sus propios límites, por ello su identidad es fortalecida desde la formación teológica brindada entablando una conversación abierta, respetuosa y razonable con todos los estudiantes de la institución.

Es claro que, si la pregunta de la investigación estaba relacionada con las formas de enseñanza, ellas juegan un papel importante en la medida que le permiten al estudiante ser realmente aprendientes, que significan desde la vida y los contextos propios los contenidos brindados por los docentes.

Ahora bien, el diálogo entre la praxis científica con los saberes humanos y teológicos requiere de mayores espacios de encuentro, los cuales deben ir más allá de una asignatura curricular. Aunque la investigación arroja algunos datos, desde los espacios ajenos al proceso pedagógico específico de la enseñanza de la teología, se alcanza a percibir desconocimiento de este proceso pedagógico por parte de algunas instancias. (Esto sería objeto de otro proceso de investigación que permita ver en forma sistémica los procesos y alcances de la formación teológica curricular en la institución).

Se puede finalizar diciendo que, como se dijo al inicio, la enseñanza de la teología se convierte entonces, en la mejor forma de mostrar como el cristianismo cree en un Dios que se ha hecho legible en la historia y audible en el mundo que exige estar en contacto con los movimientos sociales para discernir qué de esos movimientos es compatible con el Evangelio y qué no lo es, en un profundo compromiso con la vida de su audiencia y el objeto de su reflexión.

\section{Reconocimientos}

El presente artículo hace parte de la investigación "Prácticas de enseñanza en la formación teológica en contexto universitario. Estudio de caso múltiple en universidades de fundación e inspiración católica de Bogotá y Cali" El caso analizado es la Pontificia Universidad Javeriana de Cali, como una de las universidades trabajadas en la Investigación. Investigación patrocinada y financiada por las Instituciones: Pontificia Universidad Javeriana, Bogotá. Pontificia Universidad Javeriana, Cali. Fundación Universitaria Católica Lumen Gentium. Grupos de investigación: Didaskalia. De Humanitate y Yeshúa.

Todos los datos relacionados con la investigación (audios de las entrevistas y sus correspondientes transcripciones), están sujetos a un acuerdo de confidencialidad. El Editor General, de Cuadernos de Teología de la Universidad Católica del Norte, da fe de la existencia de aquellos. 


\section{Referencias Bibliográficas}

Agudelo G., D., Carrasquilla, J., \& Rojas, L. (2004). Teología: su epistemología y los nuevos paradigmas. Theologica Xaveriana, (151), 449-468. https://bit.ly/2KRU0dG

Díaz Barriga, F. (2006). Enseñanza situada: vínculo entre la escuela y la vida. México, DF: Mc Graw-Hill. https://bit.ly/3phTa99

Comisión Europea, Dirección General de Educación y Cultura. [DG EAC](2000). Informe europeo sobre la calidad de la educación escolar: Dieciséis indicadores de calidad (Educación Desarrollo de las políticas educativas, Inf.). https://bit.ly/3mEkXPq

Francisco. Vaticano II. Evangelii Gaudium.24 de noviembre de 2013. https://bit.ly/37lqKz6

Francisco. Vaticano II. Carta Encíclica Laudato Si'. 24 de mayo de 2015. https://bit.ly/3nK9BuE

Fernández, A. (2007). Los idiomas del aprendiente: análisis de modalidades de enseñanza en familias, escuelas y medios (Psicología contemporánea). Buenos Aires: Nueva Visión.

Gutiérrez, F., y Prado, C. (2015). Ecopedagogía y ciudadanía planetaria (3a ed.). Ciudad de México: De La Salle Editores.

Grupo Didaskalia. (2019). Informe del procesamiento de la herramienta Atlas Ti: Caso: Pontificia Universidad Cali. En: Prácticas de enseñanza en la formación teológica en contexto universitario. Estudio de caso múltiple en universidades de fundación e inspiración católica de Bogotá y Cali [Proyecto de investigación]. Bogotá: Pontificia Universidad Javeriana.

Habermas, J., Taylor, C., Butler, J., y West, C. (2011). El poder de la religión en la esfera pública (Estructuras y procesos. Filosofía). (J. M. Carabante y R. Serrano, Trad.). Madrid: Trotta.

Juan Pablo II. Vaticano II. Constitución apostólica Sapientia Christiana. 29 de abril de 1979. https://bit.ly/2LTfp6P

Ladriere, J. (1996). L' espérance de la raison. En J. Greisch \& G. Florival (Eds.), Création et évènement autour de Jean Ladrière: Actes de la décade du 21 au 31 août 1995, Centre international de Cerisy-la-Salle (Vol. 45, Bibliothèque philosophique de Louvain, pp. 361-387). Louvain: Éd. de l'Institut supérieur de philosophie.

Santrock, J. W. (2002). Psicología de la educación (L. E. Pineda Ayala, Trad.). México, DF: McGraw-Hill.

Treek Nilsson, M. D. V. (Abril 7, 2015). La teología en la universidad. El Mostrador. Blogs Y Opinión. https://bit.ly/38vuW4n

Zuleta Salas, G. L. (2013). Teología y Universidad [Editorial]. Cuestiones Teológicas, 40(94), 283-286. https://bit.ly/2KxMbtR

\section{Para citar este artículo bajo Norma APA 7a ed. \\ Rojas Cadena, L. (2020). Finalidad y logro de la educación teológica en un mundo plural: una mirada reflexiva en caso de estudio. Cuadernos de teología - Universidad Católica del Norte (En línea), 12, e4636, https://doi.org/10.22199/issn.0719-8175- 2020-0010}

Copyright del articulo: @2020 Leonardo Rojas

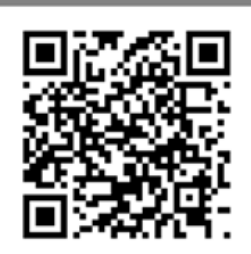

DOI

(cc) BY

Este es un artículo de acceso abierto, bajo licencia Creative Commons BY 4.0. 